\title{
Effect of Non-linear Electrodynamics on Weak field deflection angle by Black Hole
}

\author{
Wajiha Javed, ${ }^{1, *}$ Ali Hamza, ${ }^{1, \dagger}$ and Ali Övgün ${ }^{2,3, \ddagger}$ \\ ${ }^{1}$ Division of Science and Technology, University of Education, Township-Lahore, Pakistan \\ ${ }^{2}$ Instituto de Física, Pontificia Universidad Católica de Valparaíso, Casilla 4950, Valparaíso, Chile. \\ ${ }^{3}$ Physics Department, Arts and Sciences Faculty, Eastern Mediterranean University, Famagusta, North Cyprus via Mersin 10, Turkey.
}

(Dated: November 12, 2019)

\begin{abstract}
In this work, we investigate the weak deflection angle of light from exact black hole within the non-linear electrodynamics. First we calculate the Gaussian optical curvature using the optical spacetime geometry. With the help of modern geometrical way popularized by Gibbons and Werner, we examine the deflection angle of light from exact black hole. For this desire, we determine the optical Gaussian curvature and execute the Gauss-Bonnet theorem on optical metric and calculate the leading term of deflection angle in the week limit approximation. Furthermore, we likewise study the plasma medium's effect on weak gravitational lensing by exact black hole. Hence we expose the effect of the non-linear electrodynamics on the deflection angle in the week gravitational field.
\end{abstract}

PACS numbers: 95.30.Sf, 98.62.Sb, 97.60.Lf

Keywords: Weak gravitational lensing; Asymptotically flat black hole; Deflection angle; non-linear electrodynamics; Gauss-Bonnet theorem

\section{INTRODUCTION}

In 1783, accepting the corpuscular theory of light suggested by Newton which hypothesized that light comprises of small discrete particles, John Michell proposed the presence of dark stars. Michell posted a letter to Philosophical affair of the Royal Society of London [1] wherein he logically said that these small discrete particles of light are brake by the star gravitational acceleration when emitted by a star, and believed that it may be within reach to measure the star's mass dependent on the lighten in their rates. Then again, gravitational pull of a star may be very solid even the speed of light could not escape from it, then this type of star is called dark or not visible star. Michell evaluated that might be the situation when a star having size 500 times greater than the Sun's size. Michell likewise declared that space experts may identify the dark stars by studying star system gravitation-ally acting as binary stars, yet here just one star could be watched. Michell's thought went ignored for over a period of 100 years, since this was accepted that gravity couldn't be associate with light.

Moreover, in 1915, in theory of general relativity (GR), Einstein announced that gravitational lens [dispersion of matter (for example a group of galaxies) within the light origin and the viewer] could deviate the light from the light origin as the light goes near to the viewer. This impact is called gravitational lensing a guess later accepted by a test $[2,3]$ in 1919. A gravitational lensing issue gathers a well developed theory and a wide scope of observational phenomena related with the deflection of light rays with the gravity. The gravitational lens theory mostly handle with geometrical optics in vacuum and uses the idea of the deflection angle. The essential supposition that is the approximation of a weak deflection angle of a photon. General relativity announced that a light beam going close to a circular body of mass $M$ along a huge impact parameter $b$ is distracted with a little angle,

$$
\Theta=\frac{2 R_{S}}{b}=\frac{4 M}{b}, G=c=1 .
$$

This interpretation is solid if $b \succcurlyeq R_{S}$, where $R_{S}=2 M$ is the Schwarzschild radius of the gravitating body. Deflection angle (1) is typically called the "Einstein angle". In most astrophysical circumstances associated with gravitational lensing, the approximation of weak deflection is well fulfilled. The directions of photons in vacuum, just as the deflection angles, don't rely upon the light frequency, so gravitational lensing in vacuum is neutral.

It was John Wheeler who authored the word black hole $(\mathrm{BH})$ and introduced the name of wormholes and contended around the idea of reality with Einstein and Bohr [4]. Since 1919, which is the time of the exploratory checking of the deviation of light, various examinations on the gravitational lensing have been made for the $\mathrm{BHs}$ as well as for the

\footnotetext{
*Electronic address: wajiha.javed@ue.edu.pk; wajihajaved84@yahoo.com

† Electronic address: alihamza.ahg@gmail.com

${ }^{\ddagger}$ Electronic address: ali.ovgun@pucv.cl
} 
other astrophysical items ([5]-[10]).

Gravitational lensing is a helpful instrument of astrophysics [11] and astronomy, in gravitational lensing light beams from distant stars and galaxies are deviate by a planet, a BH or dark matter [12, 13]. The discovery of dark matter filaments [14] with the help of week deflection is an extremely to the point topic since it is very helpful in studying the structure of the universe [15]. From a hypothetical viewpoint, new techniques have been proposed to compute deflection angle. In 2008, Gibbons and Werner (GW) arises with another plan to calculate the deflection angle of photon [16]. Gibbons and Werner imagined that both light origin and observer lies in the asymptotic Minkowski area. In the sequel, they utilized the Gauss-Bonnet theorem (GBT) to a spatial space, which is characterized by the optical metric [16]. In GBT, we can utilize a space $D_{R}$, which is limited by the photon beam just as a circular boundary curve $C_{R}$ that is situated at focus on the focal point where the photon beam meets the light origin and observer. It is expected that both light origin and observer are at the co-ordinate length $\mathrm{R}$ from the focal point. In the week field approximation the GBT is stated in the form of optical metric as pursues [16]:

$$
\iint_{D_{R}} \mathcal{K} d S+\oint_{\partial D_{R}} \kappa d t+\Sigma_{i} \theta_{i}=2 \pi \mathcal{X}\left(D_{R}\right) .
$$

Where $\mathcal{K}$ denotes the optical Gaussian curvature and dS denotes an areal component. Subsequently thinking about the Euler characteristic $\mathcal{X}\left(D_{R}\right)=1$ also added in the jump angles $\Sigma_{i} \theta_{i}=\pi$, the deflection angle is calculated by utilizing the describing condition behaving in consistence with the straight line approximation:

$$
\alpha=-\int_{0}^{\pi} \int_{\frac{b}{r \sin \phi}}^{\infty} \mathcal{K} d S .
$$

Where deflection angle is denoted by $\Theta$. A short time later, Werner expanded this strategy for stationary BHs [17]. Next, Ishihara et al [18]. demonstrated it, this is achievable to calculate deflection angle for the finite distances (huge impact parameter) as the GW just calculated the deflection angle of BH's spacetime for the observer at asymptotically flat zone in the weak field limits utilizing the optical Fermat geometry. As of late, Crisnejo and Gallo has examined the deflection of light within the plasma medium [19]. From that point forward, there is a constantly developing concern to the weak gravitational lensing by means of the strategy used by GW named as GBT for BHs, cosmic strings either wormholes ([20]-[50]).

The primary point of this calculation is to explore the impact of the NLE on the deflection angle of exact BH and utilize the GBT wherein the deviation of light turn into a global effect. Since we just center the non-singular field outer of a light beams. We mostly examined the gravitational singularities inside the general relativity. Here, density clearly ends up unending at the origin of a $\mathrm{BH}$ and inside astronomy and cosmology as the soonest condition of the cosmos during the Big Bang. In theory of (GR), spacetime singularities rise various issues, both scientific and physical $[51,53]$. Utilizing the NLE its conceivable to resolve these singularities by calculating a regular BH solution ([54][58]). Freshly, Kruglov suggested another model of NLE with two parameters $\beta$ and $\gamma$, where the particular scope of magnetic field, the unitary standards and causality are fulfilled [59]. Furthermore, AN Aliev et al. demonstrated the impact of the magnetic field on the BH spacetime [60, 61].

This work is composed as pursues. In Sect. 2, we quickly survey the arrangement of exact $\mathrm{BH}$ and after that we compute its optical metric and the Gaussian optical curvature. In Sect. 3, deflection angle of light utilizing the GBT is computed for exact BH. In sect. 4, we observe the graphical behaviour of deflection angle in non-plasma medium. In Sect. 5, we examination the effect of plasma medium on gravitational lensing. In Sect. 6, we analyzed the graphical behaviour in presence of plasma medium. Furthermore, we finishes up in Sect. 7 with a dialog in regards to the outcomes got from the present work.

\section{EXACT OPTICAL METRIC WITH NON-LINEAR ELECTRODYNAMICS}

The activity of non-linear electrodynamics speculations which are negligibly coupled to gravity, is characterized as [62]

$$
S=\frac{1}{16 \pi} \int \sqrt{-g}(R+K(\psi)) d^{4} x
$$

where

$$
\psi=F_{\mu \nu} F^{\mu \nu}, \quad F_{\mu \nu}=\nabla_{\mu} A_{\nu}-\nabla_{\nu} A_{\mu} .
$$


Here, $R$ is Ricci scalar, $A_{\mu}$ is the Maxewell field, $g$ is gravity and $K(\psi)$ is defined as function of $\psi$. The deviation of the motion with respect to the field $A_{\mu}$ gives the derived Maxwell equation.

$$
G_{\mu \nu}=-2 K,_{\psi} F_{\mu \lambda} f_{\nu}^{\lambda}+\frac{1}{2} g_{\mu \nu} K, \quad K_{, \psi} \equiv \frac{d K}{d \psi}
$$

and

$$
\nabla_{\mu}\left(K, \psi F^{\mu \nu}\right)=0
$$

Presently, the measurement of static and spherical symmetric spacetime with the wellspring of non-linear electrodynamics field is defined as [62]

$$
d s^{2}=-U(r) d t^{2}+\frac{d r^{2}}{U(r)}+r^{2} d \Omega_{2}^{2}
$$

where

$$
U(r)=1-\frac{2 M}{r}+\frac{Q^{2}}{r^{2}}-\frac{r^{2} \alpha^{2}}{3}+2 Q \alpha, \quad d \Omega_{2}^{2}=d \theta^{2}+\sin ^{2} \theta d \phi^{2},
$$

here black hole mass is denoted by $M$, charge is denoted by $Q$ and $\alpha$ is the coupling constant. Now put the value of $U$ in Eq. (5), we get the following

$$
\begin{aligned}
d s^{2} & =-\left(1-\frac{2 M}{r}+\frac{Q^{2}}{r^{2}}-\frac{r^{2} \alpha^{2}}{3}+2 Q \alpha\right) d t^{2}+\left(1-\frac{2 M}{r}+\frac{Q^{2}}{r^{2}}\right. \\
& \left.-\frac{r^{2} \alpha^{2}}{3}+2 Q \alpha\right)^{-1} d r^{2}+r^{2} d \theta^{2}+r^{2} \sin ^{2} \theta d \phi^{2} .
\end{aligned}
$$

By accepting that light origin and onlooker lies in the tropical plane similarly direction of the null photon is additionally in a similar plane having $\left(\theta=\frac{\pi}{2}\right)$. Now, for null geodesics we put $d s^{2}=0$ and we get the following optical metric as

$$
d t^{2}=\frac{d r^{2}}{\left(1-\frac{2 M}{r}+\frac{Q^{2}}{r^{2}}-\frac{r^{2} \alpha^{2}}{3}+2 Q \alpha\right)^{2}}+\frac{r^{2} d \phi^{2}}{1-\frac{2 M}{r}+\frac{Q^{2}}{r^{2}}-\frac{r^{2} \alpha^{2}}{3}+2 Q \alpha}
$$

Now, optical metric in shape of new coordinates $r^{\star}$ is written as

$$
d t^{2}=\bar{g}_{a b} d x^{a} d x^{b}=d r^{\star 2}+f^{2}\left(r^{\star}\right) d \phi^{2}
$$

here

$$
\begin{aligned}
r^{\star} & =\frac{r}{1-\frac{2 M}{r}+\frac{Q^{2}}{r^{2}}-\frac{r^{2} \alpha^{2}}{3}+2 Q \alpha}, \\
f\left(r^{\star}\right) & =\frac{r}{\sqrt{\left(1-\frac{2 M}{r}+\frac{Q^{2}}{r^{2}}-\frac{r^{2} \alpha^{2}}{3}+2 Q \alpha\right)}} .
\end{aligned}
$$

Here we see that $(a, b)$ is converted into $(r, \phi)$ and its determinant is $\operatorname{det} \bar{g}_{a b}=\frac{1}{f(r \star)^{2}}$. Now by using Eq. (8), the non-zero christofell symbols are defined as

$$
\Gamma_{\phi \phi}^{r^{\star}}=-f\left(r^{\star}\right) f^{\prime}\left(r^{\star}\right) \text { and } \Gamma_{r^{\star} \phi}^{\phi}=\frac{f^{\prime}\left(r^{\star}\right)}{f\left(r^{\star}\right)}
$$

and the only non-vanishing Reimann tensor for optical curvature is given as $R_{r^{\star} \phi r^{\star} \phi}=-k f^{2}\left(r^{\star}\right)$ where $R_{r^{\star} \phi r^{\star} \phi}=g_{r^{\star} r^{\star}} R_{\phi r^{\star} \phi}^{r^{\star}}$. Now, Gaussian optical curvature is written as

$$
\mathcal{K}=\frac{R_{r^{\star} \phi r^{\star} \phi}}{g_{r^{\star} \phi}}=-\frac{f^{\prime \prime}\left(r^{\star}\right)}{f\left(r^{\star}\right)}=\frac{-1}{f\left(r^{\star}\right)} \frac{d^{2} f\left(r^{\star}\right)}{d r^{\star 2}} .
$$


With the help of previous equation, we can say that the intrinsic Gaussian optical curvature denoted by $\mathcal{K}$ is written in expression of $r$

$$
\mathcal{K}=\frac{-1}{f\left(r^{\star}\right)}\left[\frac{d r}{d r^{\star}} \frac{d}{d r}\left(\frac{d r}{d r^{\star}}\right) \frac{d f}{d r}+\frac{d^{2} f}{d r^{2}}\left(\frac{d r}{d r^{\star}}\right)^{2}\right] .
$$

Finally now we calculate the relevant Gaussian optical curvature for exact BH by putting the Eq. (9) into Eq. (11), we calculate the suite formulation

$$
\begin{aligned}
\mathcal{K} & =\frac{-2 M}{r^{3}}\left(1-\frac{3 M}{2 r}\right)+\frac{3 Q^{2}}{r^{4}}\left(1+\frac{2 Q^{2}}{3 r^{2}}\right)-\frac{4 M Q \alpha}{r^{3}}-\frac{6 M Q^{2}}{r^{5}} \\
& -\frac{2 Q^{2} \alpha}{r^{2}}\left(\alpha-\frac{3 Q}{r^{2}}\right)+\alpha^{2}\left(\frac{2 M}{r}-\frac{1}{3}-\frac{2 Q \alpha}{3}\right)
\end{aligned}
$$

and so, it can be written as

$$
\mathcal{K}=\frac{-2 M}{r^{3}}+\frac{3 Q^{2}}{r^{4}}-\frac{4 M Q \alpha}{r^{3}}+\mathcal{O}\left(M^{2}, Q^{3}, \alpha^{2}\right)
$$

\section{DEFLECTION ANGLE OF EXACT BLACK HOLE WITHIN THE NON-LINEAR ELECTRODYNAMICS}

Now with the help of Gauss-Bonnet theorem we derive the deflection angle of a exact black hole in the presence of non-linear electrodynamics. We apply the Gauss-Bonnet theorem to the region $\mathcal{D}_{R}$, stated as [16]

$$
\iint_{\mathcal{D}_{R}} \mathcal{K} d S+\oint_{\partial \mathcal{D}_{R}} k d t+\sum_{i} \epsilon_{i}=2 \pi \mathcal{X}\left(\mathcal{D}_{R}\right)
$$

where Gaussian curvature is denoted by $\mathcal{K}$ and geodesic curvature is denoted by $k$, stated as $k=\bar{g}\left(\nabla_{\dot{\gamma}} \dot{\gamma}, \ddot{\gamma}\right)$ in such a way that $\bar{g}(\dot{\gamma}, \dot{\gamma})=1$, here $\ddot{\gamma}$ is the representation for unit acceleration vector and the $\epsilon_{i}$ is the corresponding exterior angle at the ith vertex. As $R \rightarrow \infty$, both the jump angles become $\pi / 2$ and we obtained $\theta_{O}+\theta_{S} \rightarrow \pi$. The Euler characteristic is $\mathcal{X}\left(\mathcal{D}_{R}\right)=1$, as $\mathcal{D}_{R}$ is non singular. Therefore we get

$$
\iint_{\mathcal{D}_{R}} \mathcal{K} d S+\oint_{\partial \mathcal{D}_{R}} k d t+\epsilon_{i}=2 \pi \mathcal{X}\left(\mathcal{D}_{R}\right)
$$

here, $\epsilon_{i}=\pi$ proves that $\gamma_{\bar{g}}$ and the total jump angle is a geodesic, since the Euler characteristic number denoted by $\mathcal{X}$ is 1 . As $R \rightarrow \infty$, the only interesting part to be calculated is $k\left(C_{R}\right)=\left|\nabla_{\dot{C}_{R}} \dot{C}_{R}\right|$. Since, geodesic curvature's radial component is given by [16]

$$
\left(\nabla_{\dot{C}_{R}} \dot{C}_{R}\right)^{r}=\dot{C}_{R}^{\phi} \partial_{\phi} \dot{C}_{R}^{r}+\Gamma_{\phi \phi}^{r^{*}}\left(\dot{C}_{R}^{\phi}\right)^{2}
$$

For large $R, C_{R}:=r(\phi)=R=$ constant. Hence, the form of the equation Eq. (16) becomes $\left(\dot{C}_{R}^{\phi}\right)^{2}=\frac{1}{f^{2}\left(r^{\star}\right)}$. Remembering $\Gamma_{\phi \phi}^{r^{\star}}=-f\left(r^{\star}\right) f^{\prime}\left(r^{\star}\right)$, it becomes

$$
\left(\nabla_{\dot{C}_{R}^{r}} \dot{C}_{R}^{r}\right)^{r} \rightarrow \frac{-1}{R} .
$$

Hence it given that the topological defect is not involved in the geodesic curvature. So, $k\left(C_{R}\right) \rightarrow R^{-1}$. But with the help of optical metric Eq. (8), we can write as $d t=R d \phi$. Hence, we came up;

$$
k\left(C_{R}\right) d t=\frac{1}{R} R d \phi .
$$

Combining all the above results, we have

$$
\iint_{\mathcal{D}_{R}} \mathcal{K} d s+\oint_{\partial \mathcal{D}_{R}} k d t={ }^{R \rightarrow \infty} \iint_{S_{\infty}} \mathcal{K} d S+\int_{0}^{\pi+\Theta} d \phi .
$$


The light ray in the weak deflection limit at 0th order is defined as $r(t)=b / \sin \phi$. So with the help of Eq. (13) and Eq. (20), the deflection angle defined as [16]

$$
\Theta=-\int_{0}^{\pi} \int_{b / \sin \phi}^{\infty} \mathcal{K} \sqrt{\operatorname{det} \bar{g}} d r^{\star} d \phi
$$

where

$$
\sqrt{\operatorname{det} \bar{g}}=r\left(1-\frac{3 M}{r}+\frac{3 Q^{2}}{2 r^{2}}+3 Q \alpha\right) d r
$$

After putting the leading order terms of Gaussian curvature Eq. (13) into Eq. (20), the deflection angle is defined as:

$$
\Theta \approx \frac{4 M}{b}-\frac{3 \pi Q^{2}}{4 b^{2}}+\frac{20 M Q \alpha}{b}+\mathcal{O}\left(M^{2}, Q^{3}, \alpha^{2}\right) .
$$

\section{GRAPHICAL ANALYSIS FOR NON-PLASMA MEDIUM}

This area is concerned to talk about the graphical behavior of the deflection angle. We also talk about the physical importance of above mentioned plots and observe the effect of coupling constant $\alpha$, impact parameter $b$ and $\mathrm{BH}$ charge $Q$ on deflection angle.

\section{A. Deflection angle $\Theta$ w.r.t Coupling constant $\alpha$}

(i) $b=5$

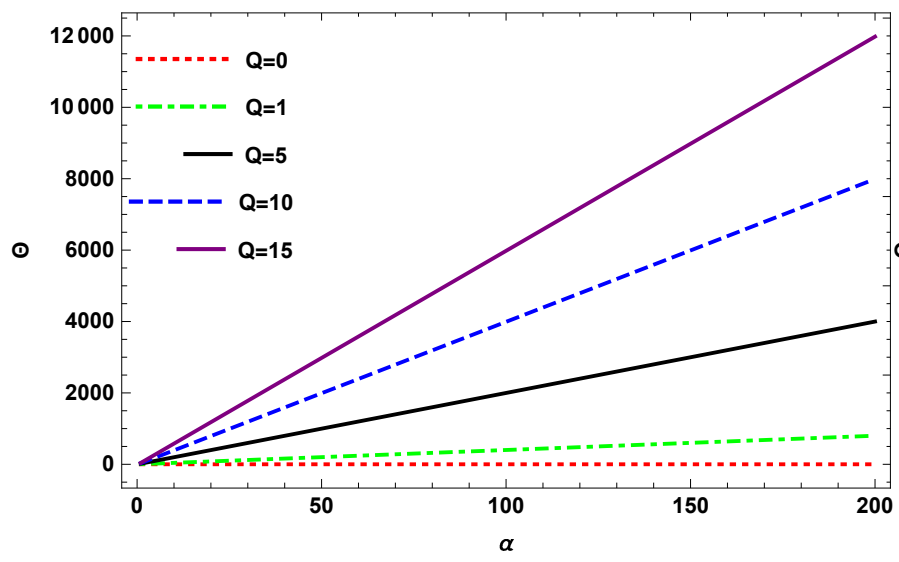

(ii) $Q=5$

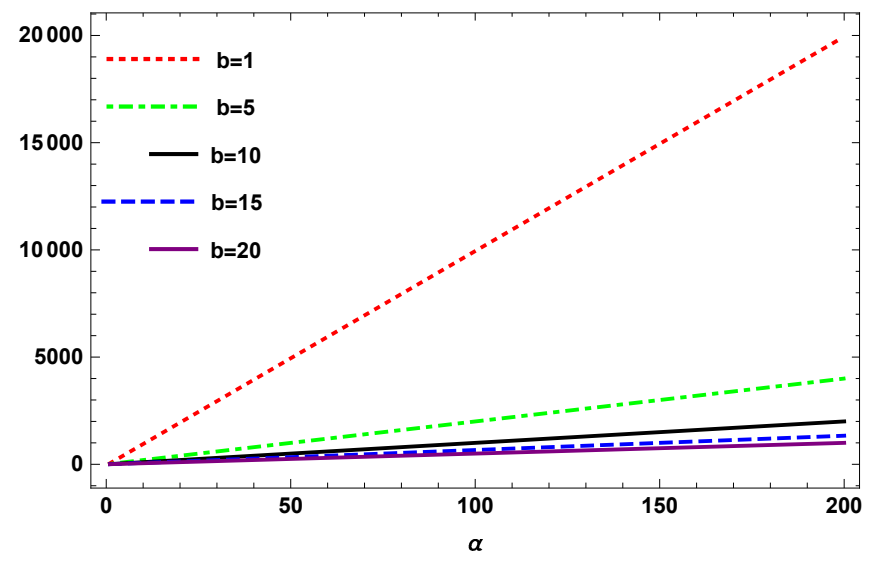

Figure 1: Relation between $\Theta$ and $\alpha$.

- Figure 1 shows the observance of $\Theta$ w.r.t $\alpha$ by varying $Q$ and set $b=5$ and vary the value of $b$ and fixed $Q=5$ respectively.

1. In plot (i), we observed that $\Theta$ gradually decreasing for large values $Q$.

2. In plot (ii), we noted that $\Theta$ is gradually increasing for large values $b$. 


\section{B. Deflection angle $\Theta$ w.r.t Impact parameter $b$}
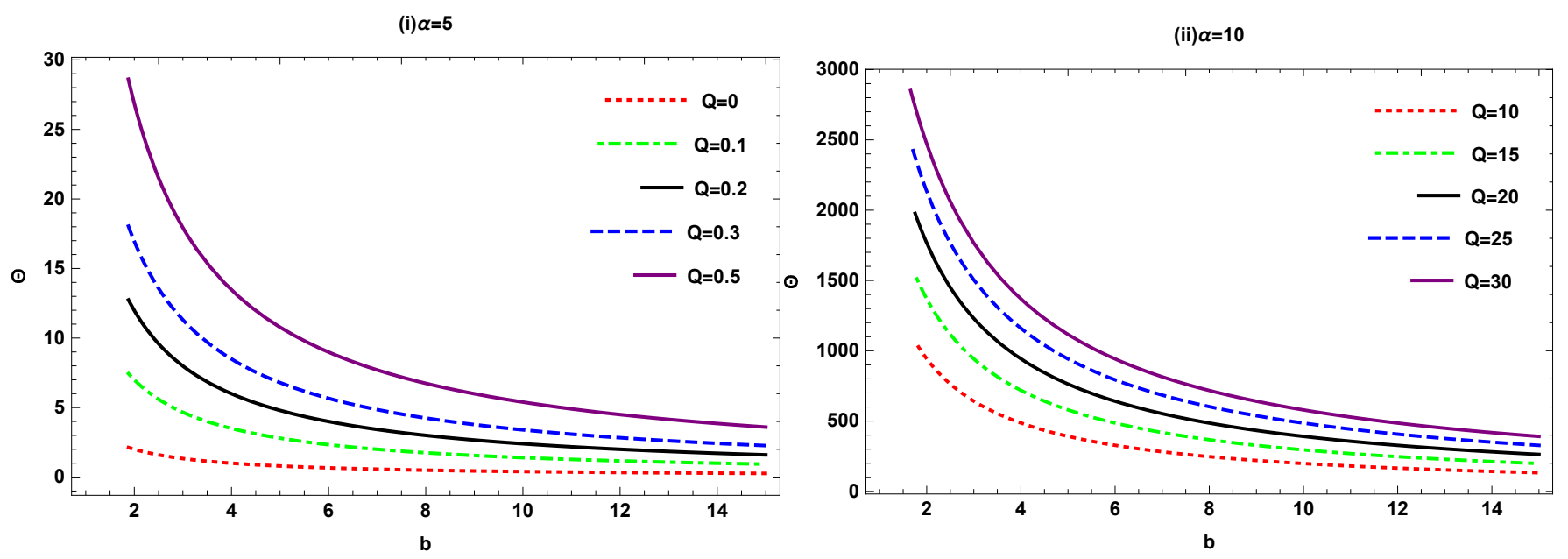

Figure 2(a): Relation between $\Theta$ and $b$.

- Figure 2(a) displays the observance of $\Theta$ w.r.t $b$ by taking $\alpha$ fixed and $Q$ changing.

1. In figure (i)and (ii), we analyzed that $\Theta$ gradually decreasing for both small and large values of $Q$.

(i) $Q=0.45$

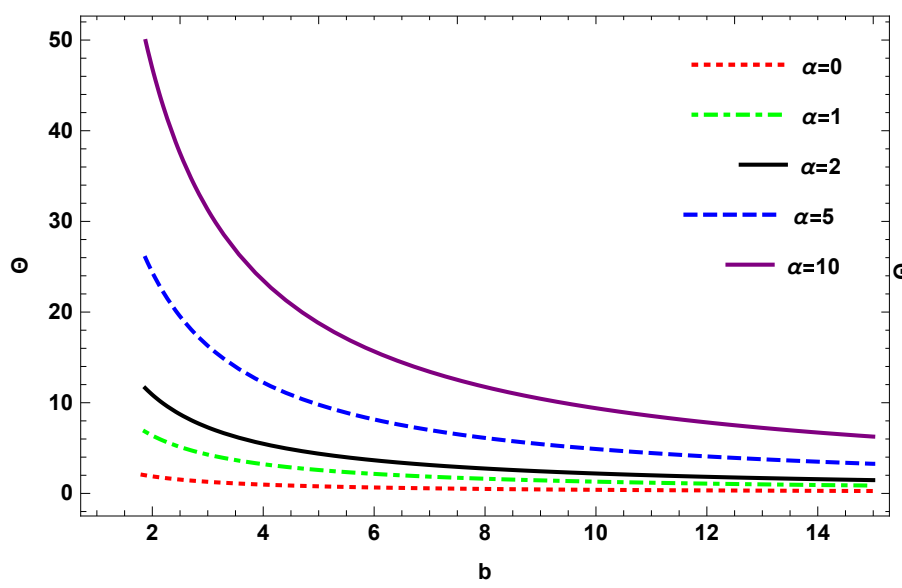

(ii) $Q=10$

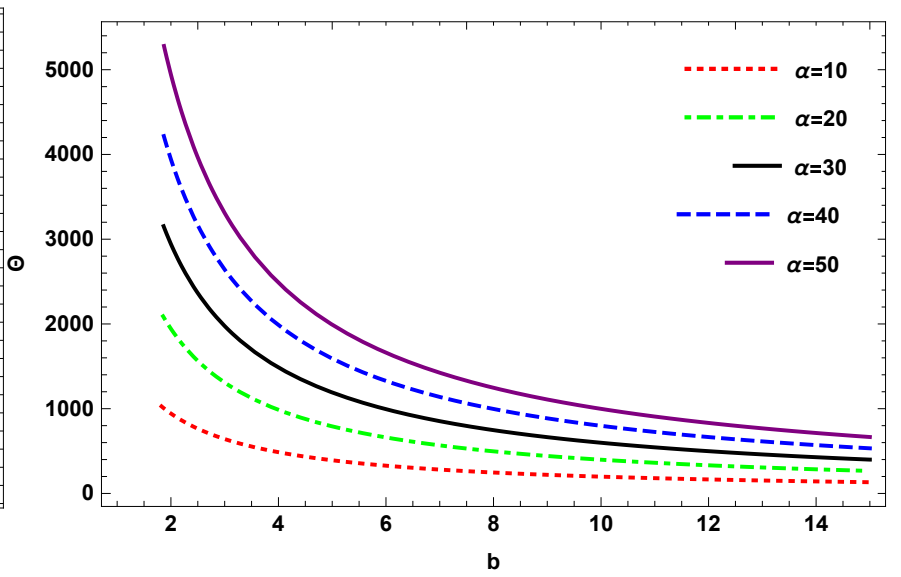

Figure 2(b): Relation between $\Theta$ and $b$.

- Figure 2(b) shows the observance of $\Theta$ w.r.t $b$ by taking the BH charge fixed and varying the coupling constant.

1. In plot(i) and (ii), we analyze that $\Theta$ is also gradually reducing for both large and small values of coupling constant. 


\section{Deflection angle $\Theta$ w.r.t BH Charge $Q$}

(i) $\alpha=5$

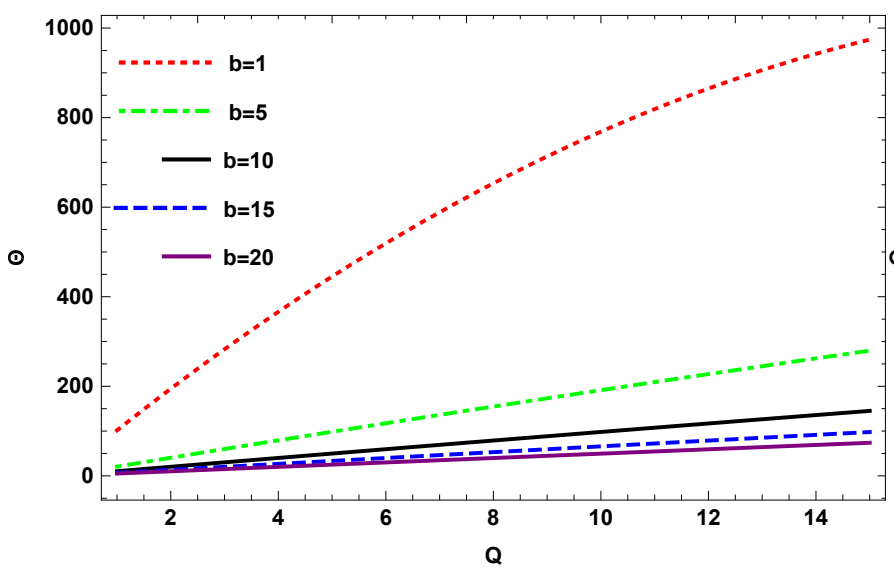

(ii) $b=5$

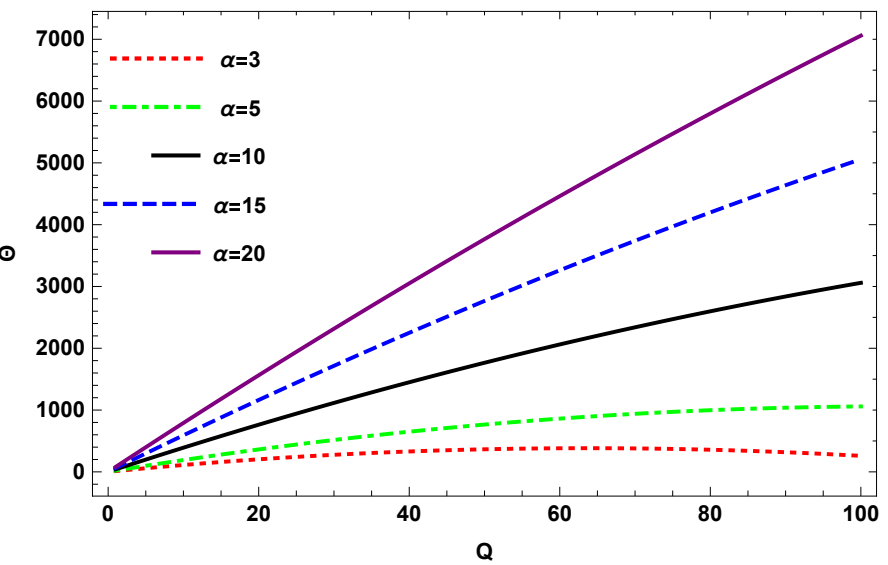

Figure 3: Relation between $\Theta$ and $Q$.

- Figure 3 depict the observance of $\Theta$ w.r.t $Q$ for setting $\alpha=5$ and changing $b$ and for setting $b=5$ and varying $\alpha$ respectively.

1. In picture (i), we analyzed that $\Theta$ exponentially increasing for large values of impact parameter and deflection angle rapidly increase for $1<b<5$.

2. In picture (ii), we examined that $\Theta$ gradually decreasing for large values of coupling constant.

\section{EFFECT OF PLASMA ON GRAVITATIONAL LENSING}

Here, we examine the impact of plasma medium on the gravitational lensing of exact BH. Let us consider a exact $\mathrm{BH}$ loaded up with plasma depicted by the refractive index $n,[19]$

$$
n^{2}(r, \omega(r))=1-\frac{\omega_{e}^{2}(r)}{\omega_{\infty}^{2}(r)} .
$$

The refractive index for this case reads

$$
n(r)=\sqrt{1-\frac{\omega_{e}^{2}}{\omega_{\infty}^{2}}\left(1-\frac{2 M}{r}+\frac{Q^{2}}{r^{2}}-\frac{r^{2} \alpha^{2}}{3}+2 Q \alpha\right)},
$$

where the metric function is defined by

$$
d s^{2}=-U(r) d t^{2}+\frac{1}{U(r)} d r^{2}+r^{2} d \Omega_{2}^{2}
$$

and

$$
U(r)=1-\frac{2 M}{r}+\frac{Q^{2}}{r^{2}}-\frac{r^{2} \alpha^{2}}{3}+2 Q \alpha .
$$

By accepting both the light origin and onlooker lies in the tropical plane similarly direction of the null photon is on a similar plane having $\left(\theta=\frac{\pi}{2}\right)$. Now, for null geodesics we put $d s^{2}=0$ and we get the following optical metric as [19]

$$
d t^{2}=g_{l m}^{o p t} d x^{l} d x^{m}=n^{2}\left[\frac{d r^{2}}{U^{2}(r)}+\frac{r^{2} d \phi^{2}}{U(r)}\right]
$$

with determinant $g_{l m}^{o p t}$,

$$
\sqrt{g^{o p t}}=r\left(1-\frac{\omega_{e}^{2}}{\omega_{\infty}^{2}}\right)+M\left(3-\frac{\omega_{e}^{2}}{\omega_{\infty}^{2}}\right)-\frac{Q^{2}}{2 r}\left(3-\frac{\omega_{e}^{2}}{\omega_{\infty}^{2}}\right)-Q \alpha r\left(3-\frac{\omega_{e}^{2}}{\omega_{\infty}^{2}}\right) .
$$


With the help of Eq. (26), we can define the non-zero christofell symbols as

and

$$
\begin{gathered}
\Gamma_{00}^{0}=\left(1+\frac{\omega_{e}^{2} A}{\omega_{\infty}^{2}}\right)\left[-A^{\prime} A^{-1}\left(1-\frac{\omega_{e}^{2} A}{\omega_{\infty}^{2}}\right)-\frac{A^{\prime} \omega_{e}^{2}}{2 \omega_{\infty}^{2}}\right] \\
\Gamma_{10}^{1}=\left(1+\frac{\omega_{e}^{2} A}{\omega_{\infty}^{2}}\right)\left[r^{-1}\left(1-\frac{\omega_{e}^{2} A}{\omega_{\infty}^{2}}-\frac{A^{\prime} A^{-1}}{2}\left(1-\frac{\omega_{e}^{2} A}{\omega_{\infty}^{2}}\right)-\frac{A^{\prime} \omega_{e}^{2}}{2 \omega_{\infty}^{2}}\right]\right.
\end{gathered}
$$

$$
\Gamma_{11}^{0}=\left(1+\frac{A \omega_{e}^{2}}{\omega_{\infty}^{2}}\right)\left[-r A\left(1-\frac{A \omega_{e}^{2}}{\omega_{\infty}^{2}}\right)+\frac{r^{2} A^{\prime}}{2}\left(1-\frac{A \omega_{e}^{2}}{\omega_{\infty}^{2}}\right)+\frac{r^{2} A}{2} \frac{A^{\prime} \omega_{e}^{2}}{\omega_{\infty}^{2}}\right]
$$

Gaussian curvature in terms of curvature tensor can be determined as

$$
\mathcal{K}=\frac{R_{r \phi r \phi}\left(g^{o p t}\right)}{\operatorname{det}\left(g^{o p t}\right)}
$$

with the help of Eq. (28) Gaussian curvature is written as

$$
\begin{aligned}
\mathcal{K} & =\frac{M}{r^{3}}\left(-2-\frac{\omega_{e}^{2}}{\omega_{\infty}^{2}}+\frac{2 \omega_{e}^{4}}{\omega_{\infty}^{4}}\right)+\frac{2 M Q^{2}}{r^{5}}\left(1-\frac{17 \omega_{e}^{2}}{\omega_{\infty}^{2}}+\frac{5 \omega_{e}^{4}}{\omega_{\infty}^{4}}\right) \\
& -\frac{4 M Q \alpha}{r^{3}}\left(1+\frac{\omega_{e}^{2}}{\omega_{\infty}^{2}}-3 \frac{\omega_{e}^{4}}{\omega_{\infty}^{4}}\right)+\mathcal{O}\left(M^{2}, Q^{3}, \alpha^{2}\right) .
\end{aligned}
$$

With the help of Gauss-Bonnet theorem we calculate the deflection angle in order to relate it with non-plasma. For calculating angle in the weak field region, as the light beams travels along a straight line approximation so used the consideration of $r=\frac{b}{\sin \phi}$ at zero order.

$$
\Theta=-\lim _{R \rightarrow 0} \int_{0}^{\pi} \int_{\frac{b}{\sin \phi}}^{R} \mathcal{K} d S
$$

With the help of Eq. (19), the deflection angle of light in plasma medium is defined as;

$$
\Theta=\frac{4 M}{b}-\frac{2 M \omega_{e}^{2}}{b \omega_{\infty}^{2}}-\frac{6 M \omega_{e}^{4}}{b \omega_{\infty}^{4}}-\frac{3 Q^{2} \pi}{4 b^{2}}+\frac{3 Q^{2} \pi \omega_{e}^{4}}{4 b^{2} \omega_{\infty}^{4}}+\frac{4 M Q \alpha}{b}+\frac{2 M Q \alpha \omega_{e}^{2}}{b \omega_{\infty}^{2}}+\mathcal{O}\left(M^{2}, Q^{3}, \alpha^{2}\right) .
$$

\section{GRAPHICAL ANALYSIS FOR PLASMA MEDIUM}

In this section our aim is to review the graphical behaviour of deflection angle in the presence of plasma medium. Here, we take $M=1, \frac{\omega_{e}}{\omega_{\infty}}=10^{-1}$ and vary the impact parameter, coupling constant and BH charge for obtaining these graphs.

\section{A. Deflection angle w.r.t Coupling constant}

(i) $b=5$

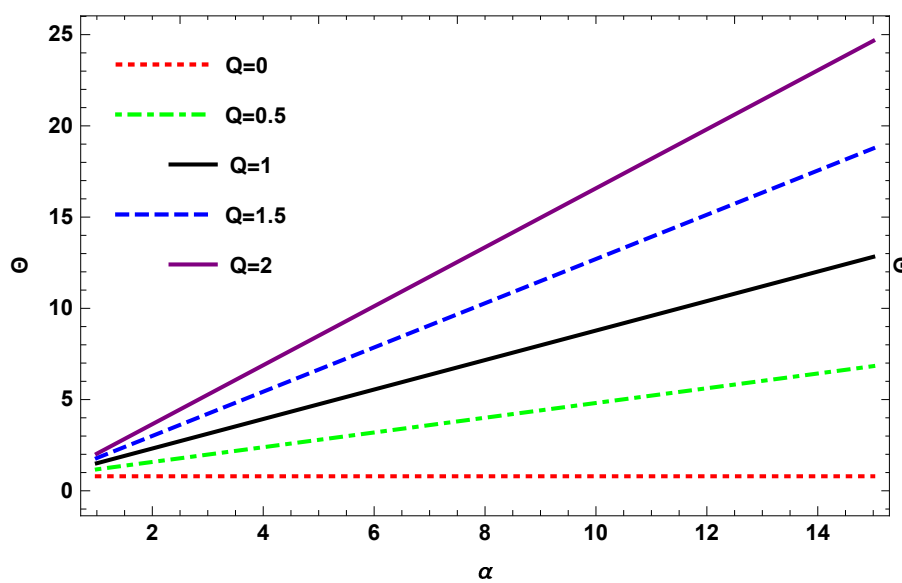

(ii) $Q=5$

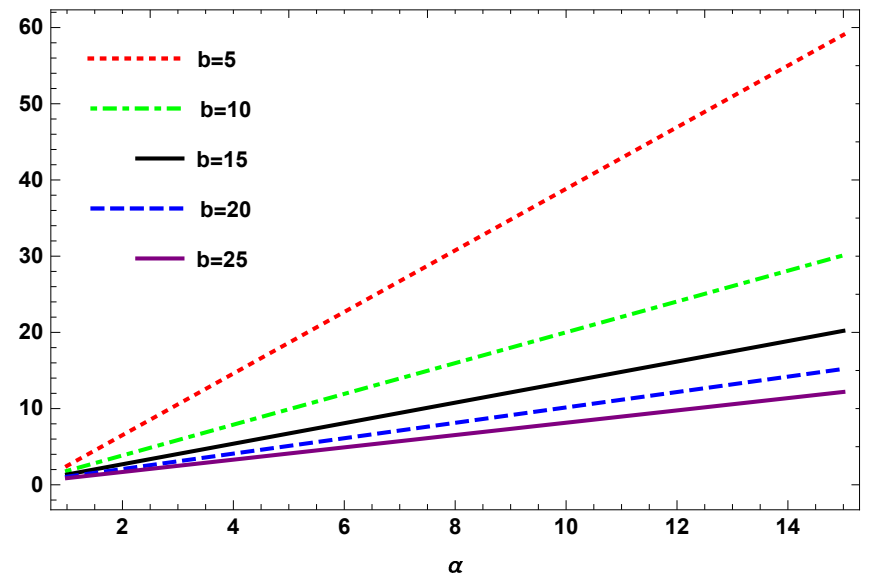


Figure 4: Relation between $\Theta$ and $\alpha$.

- Figure 4 demonstrates the behavior of $\Theta$ w.r.t $\alpha$ for setting $b=5$ and changing $Q$ and setting $Q=5$ and varying $b$ respectively.

1. In plot (i), we saw that $\Theta$ gradually decreasing for small values of $\mathrm{BH}$ charge $Q$ and graph shows the positive slope.

2. In plot (ii), we analyzed that $\Theta$ exponentially increasing for large values of $b$.

\section{B. Deflection angle $\Theta$ w.r.t Impact parameter $b$}

(i) $\alpha=5$

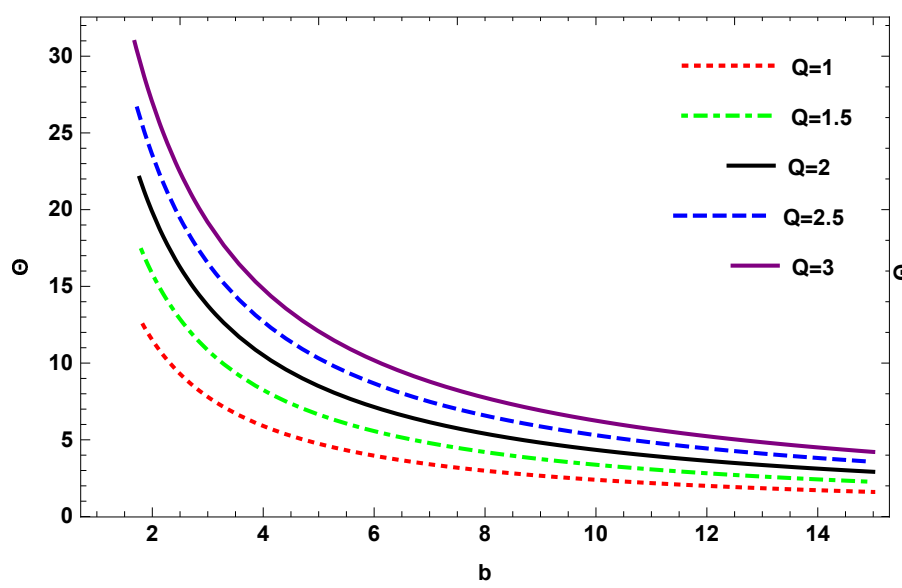

(ii) $Q=10$

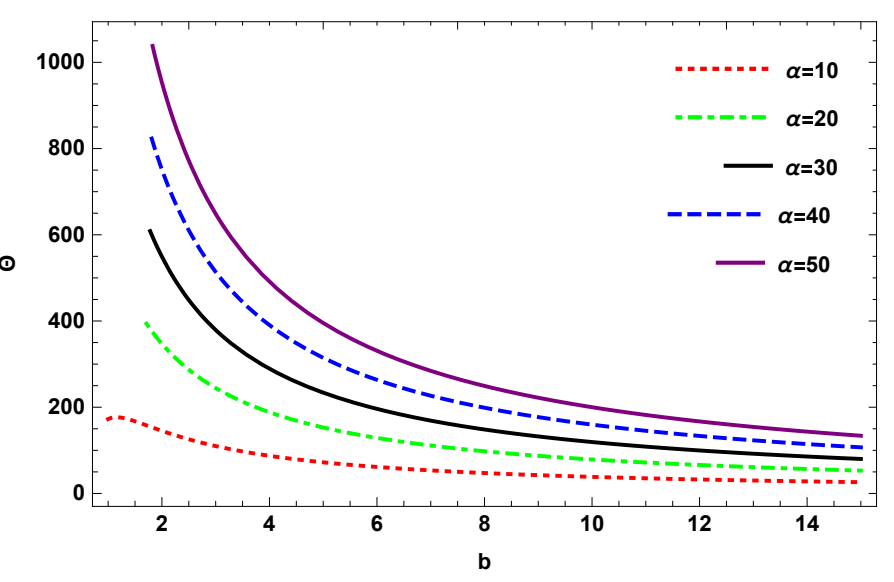

Figure 5: Relation between $\Theta$ and $b$.

- Figure 5 shows the behaviour of $\Theta$ w.r.t $b$ for setting $\alpha=5$ and changing the values of $Q$ and fixed $Q$ and varying $\alpha$ respectively.

1. In picture (i), we analyzed that $\Theta$ gradually decreasing for low values of $Q$ and then goes to positive infinity.

2. In picture (ii), we saw that $\Theta$ is gradually decreasing for large values of $\alpha$ and then goes to positive infinity.

\section{Deflection angle w.r.t Charge $Q$}

(i) $\alpha=15$

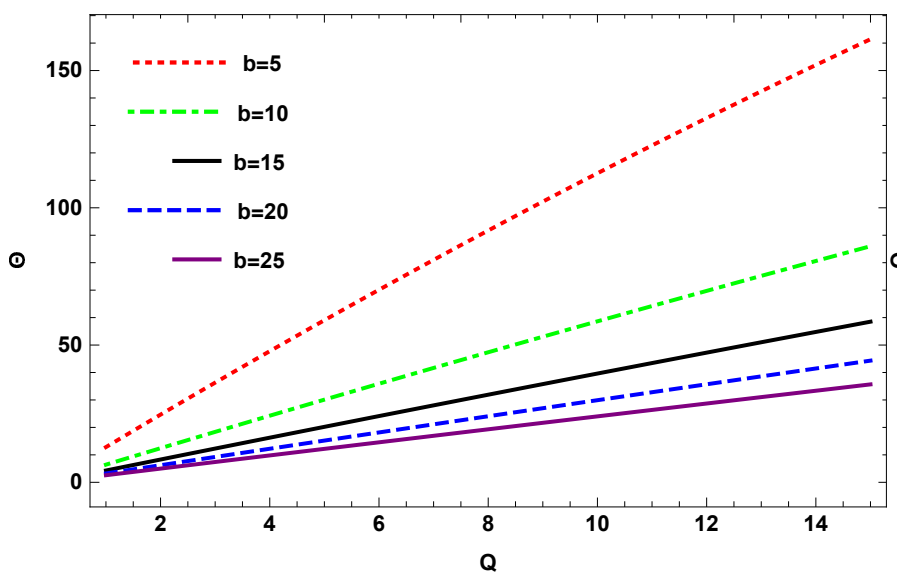

(ii) $b=5$

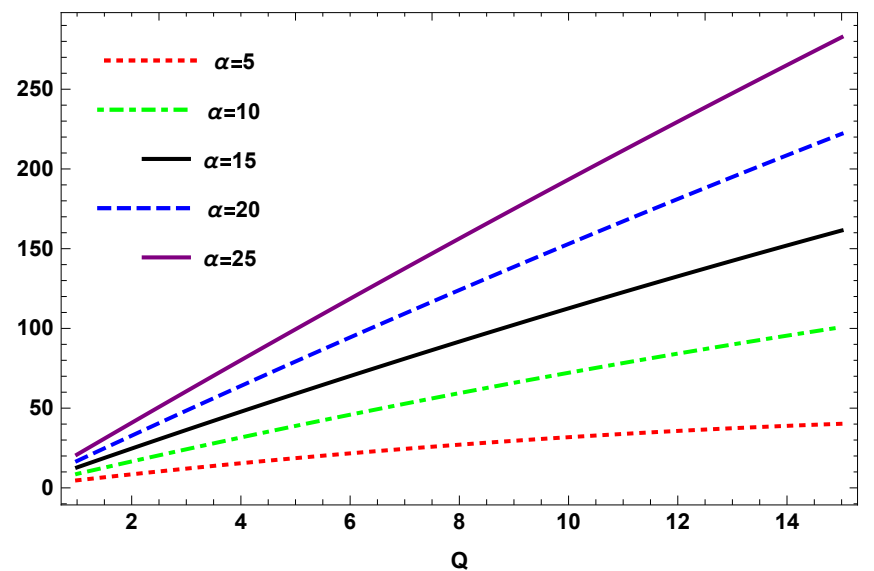

Figure 6: Relation between $\Theta$ and $Q$.

- Figure 6 shows the behaviour of deflection angle w.r.t BH charge for fixed coupling constant and varying impact parameter and fixed impact parameter and varying coupling constant respectively. 
1. In plot (i), we analyzed that $\Theta$ gradually increasing for large values of impact parameter and the behavior is positive slope.

2. In plot (ii), we saw that $\Theta$ gradually increasing for large values of coupling constant and the behavior is positive slope.

\section{SUMMARY}

In this work, we have calculated the deflection angle for exact BH in the framework of NLE. For doing this, we have used the Gauss-Bonnet theorem, and we have determined the deflection angle for exact BH with NLE. We have utilized the GBT and find the deflection angle of photons by integrating exterior of the impact parameter, that represent that gravitational lensing is a global impact and is a useful asset to analyze most of the singularities of $\mathrm{BH}$. In this calculation, we get the deflection angle of light by exact BH in the weak field limit by utilizing GBT. Hence, the deflection angle (22) is expressed as

$$
\Theta \approx \frac{4 M}{b}-\frac{3 \pi Q^{2}}{4 b^{2}}+\frac{20 M Q \alpha}{b}+\mathcal{O}\left(M^{2}, Q^{3}, \alpha^{2}\right) .
$$

By setting $\mathrm{Q}=0$ in above equation, our proposed deflection angle reduce into Schwarzschild deflection angle up to first order. We also analyze graphical behavior of deflection angle for exact BH in the background of NLE. Furthermore, we likewise computed the deflection angle of photons by exact BH with NLE in plasma medium. Deflection angle of photons in the presence of plasma medium is defined as

$$
\Theta=\frac{4 M}{b}-\frac{2 M \omega_{e}^{2}}{b \omega_{\infty}^{2}}-\frac{6 M \omega_{e}^{4}}{b \omega_{\infty}^{4}}-\frac{3 Q^{2} \pi}{4 b^{2}}+\frac{3 Q^{2} \pi \omega_{e}^{4}}{4 b^{2} \omega_{\infty}^{4}}+\frac{4 M Q \alpha}{b}+\frac{2 M Q \alpha \omega_{e}^{2}}{b \omega_{\infty}^{2}}+\mathcal{O}\left(M^{2}, Q^{3}, \alpha^{2}\right) .
$$

By neglecting the plasma impact $\left(\frac{\omega_{e}}{\omega_{\infty}} \rightarrow 0\right)$, Eq. (31) reduce into Eq. (22).

We have observed the behaviour of deflection angle w.r.t impact parameter $\mathrm{b}$, coupling constant $\alpha$ and BH charge q. The consequence found by analyze the deflection angle obtained in this paper are summed as follows:

\section{Deflection angle w.r.t Impact parameter:}

1. In our examination we observed that deflection angle gradually decrease for big values of $Q$.

2. Also we examined that deflection angle is gradually decrease for big values of $\alpha$ which shows the stability of our proposed deflection angle.

\section{Deflection angle w.r.t Coupling constant:}

1. It is to be investigated that one can only observe the stable behavior of deflection angle by exact $\mathrm{BH}$ for $0<$ $Q \leq 2$.

2. It is to be noted that the obtained deflection angle is positively increasing by increasing the impact parameter, that indicates the stable behavior.

\section{Deflection angle w.r.t BH charge:}

1. We analyzed that deflection angle exponentially increasing for large values of impact parameter.

2. We also examined that there is direct relation between deflection angle and coupling constant.

\section{Acknowledgments}

This work was supported by Comisión Nacional de Ciencias y Tecnología of Chile through FONDECYT Grant $N^{\circ}$ 3170035 (A. Ö.).

[1] S. Schaffer, Journal for the History of Astronomy 10, 42 (1979). 
[2] F.W. Dyson, A. S. Eddington, C. Davidson, "A Determination of the Deflection of Light by the Sun's Gravitational Field, from Observations Made at the Total Eclipse of May 29, 1919". Philosophical Transactions of the Royal Society A: Mathematical, Physical and Engineering Sciences. 220, 291 (1920).

[3] M. Longair, Phil. Trans. R. Soc. A 373, 20140287 (2015).

[4] C.W. Misner, K. S. Thorne, and J. A. Wheeler, Gravitation (W. H. Freeman, San Francisco, 1973).

[5] C. Darwin, Proc. R. Soc. A 249, 180 (1959).

[6] V. Bozza, Phys. Rev. D 66, 103001 (2002).

[7] S. V. Iyer and A. O. Petters, Gen. Relativ. Gravit. 39, 1563 (2007).

[8] V. Bozza, and G. Scarpetta, Phys. Rev. D 76, 083008 (2007).

[9] V. Bozza, Gen. Rel. Grav. 42, 2269 (2010).

[10] S. Frittelli, T. P. Kling, and E. T. Newman, Phys. Rev. D 61, 064021 (2000).

[11] V. Perlick, Living Rev. Rel. 7, 9 (2004).

[12] V. Bozza, Gen. Rel. Grav. 42, 2269 (2010).

[13] I. Z. Stefanov, S. S. Yazadjiev and G. G. Gyulchev, Phys. Rev. Lett. 104, 251103 (2010).

[14] S. D. Epps and M. J. Hudson, Mon. Not. Roy. Astron. Soc. 468, no. 3, 2605 (2017).

[15] M. Bartelmann and M. Maturi, arXiv:1612.06535 [astro-ph.CO].

[16] G.W. Gibbons and M. C.Werner, Class. Quant. Grav. 25, 235009 (2008).

[17] M.C. Werner, Gen. Relat. gravit. 44, 3047 (2012).

[18] A. Ishihara,Y. Suzuki,T.Ono,T. Kitamura,H.Asada, Phys. Rev. D 94(8), 084015 (2016).

[19] G. Crisnejo, E. Gallo, Phys. Rev. D 97(12), 124016 (2018).

[20] K. Jusufi, M.C. Werner, A. Banerjee, A. Ovgun, Phys. Rev. D 95(10), 104012 (2017).

[21] I. Sakalli, A. Ovgun, EPL 118(6), 60006 (2017).

[22] K. Jusufi, A. Ovgun, Phys. Rev. D 97(2), 024042 (2018).

[23] W. Javed, R. Babar, A. Ovgun, Phys. Rev. D 99(8), 084012 (2019).

[24] A. Övgün, Phys. Rev. D 99, 104075 (2019).

[25] H. Arakida, Gen. Rel. Grav. 50, 48 (2018).

[26] T. Ono, A. Ishihara and H. Asada, Phys. Rev. D 98, 044047 (2018).

[27] T. Ono, A. Ishihara and H. Asada, Phys. Rev. D 96, 104037 (2017).

[28] K. Jusufi, A. Övgün and A. Banerjee, Phys. Rev. D 96, n084036 (2017) Addendum: [Phys. Rev. D 96, 089904 (2017)].

[29] A. Övgün, K. Jusufi and I. Sakalli, Phys. Rev. D 99, 024042 (2019).

[30] K. Jusufi and A. Övgün, Int. J. Geom. Meth. Mod. Phys. (2019) 1950116.

[31] K. Jusufi, M. C. Werner, A. Banerjee, and A. Övgün, Phys. Rev. D 95, no. 10, 104012 (2017).

[32] K. Jusufi, I. Sakalli, and A. Övgün, Phys. Rev. D 96, no. 2, 024040 (2017).

[33] T. Ono, A. Ishihara, and H. Asada, Phys. Rev. D 99, no. 12, 124030 (2019).

[34] K. Jusufi, A. Övgün, A. Banerjee and I. Sakalli, Eur. Phys. J. Plus 134, no. 9, 428 (2019).

[35] G. Crisnejo, E. Gallo, and A. Rogers, Phys. Rev. D 99, 124001 (2019).

[36] G. Crisnejo, E. Gallo, and J. R. Villanueva, Phys. Rev. D 100, no. 4, 044006 (2019).

[37] A. Övgün, G. Gyulchev, and K. Jusufi, Annals Phys. 406, 152 (2019).

[38] K. Jusufi and A. Övgün, Phys. Rev. D 97, 064030 (2018).

[39] K. Jusufi, A. Övgün, J. Saavedra, Y. Vasquez, and P. A. Gonzalez, Phys. Rev. D 97, 124024 (2018).

[40] A. Övgün, Phys. Rev. D 98, 044033 (2018).

[41] A. Övgün, K. Jusufi, and I. Sakalli, Annals Phys. 399, 193 (2018).

[42] A. Övgün, Universe 5, 115 (2019).

[43] A. Övgün, I. Sakalli, and J. Saavedra, Annals Phys. 411, 167978 (2019).

[44] A. Övgün, I. Sakalli, and J. Saavedra, JCAP 1810, 041 (2018).

[45] W. Javed, R. Babar, and A. Övgün, Preprints 2019050148, 2019. (Accepted from Phys. Rev. D. https://journals.aps.org/prd/accepted/7a075Q24U941802722837310d7e657c9715a556a4)

[46] W. Javed, J. Abbas, and A. Övgün, Eur. Phys. J. C 79, no. 8, 694 (2019).

[47] W. Javed, J. Abbas and A. Övgün, Preprints 2019, 2019060124. (doi: 10.20944/preprints201906.0124.v1).

[48] W. Javed, j. Abbas and A. Övgün, Phys. Rev. D 100, no. 4, 044052 (2019).

[49] Y. Kumaran and A. Övgün, arXiv:1905.11710 [gr-qc].

[50] A. Övgün, I. Sakalli and J. Saavedra, arXiv:1908.04261 [gr-qc].

[51] S.I. Kruglov, Universe 4(5), 66 (2018).

[52] S.I.Kruglov, Ann. Phys. 529(8), 1700073 (2017).

[53] S.I. Kruglov, Ann. Phys. 383, 550 (2017).

[54] K.A. Bronnikov, Phys. Rev. D 63, 044005 (2001).

[55] E. Ayon-Beato, A. Garcia, Phys. Rev. Lett. 80, 5056 (1998).

[56] E.Ayon-Beato, A. Garcia, Gen. Relat. Gravit. 31, 629 (1999).

[57] M.E. Rodrigues, MVdS Silva, JCAP 1806(06), 025 (2018).

[58] S.A. Hayward, Phys. Rev. Lett. 96, 031103 (2006).

[59] S.I. Kruglov, Int. J. Mod. Phys. A 32(23n24), 1750147 (2017).

[60] A.N. Aliev, D.V. Galtsov, Sov. Phys. Usp. 32, 75 (1989).

[61] A.N. Aliev, Phys. Rev. D 74, 024011 (2006).

[62] S. Yu and C. Gao, arXiv:1907.00515 [gr-qc]. 